$\frac{4}{6-1491950}$

PPPL-2760

UC-421,427
PREPARED FOR THE U.S. DEPARTMENT OF ENERGY, UINDER CONTRACT DE-AC02-76-CHO-3073

PPPL-2760

TOKAMAK ERROR FIELDS AND LOCKED MODES

BY

A. REIMAN AND D. MONTICELLO

May 1991

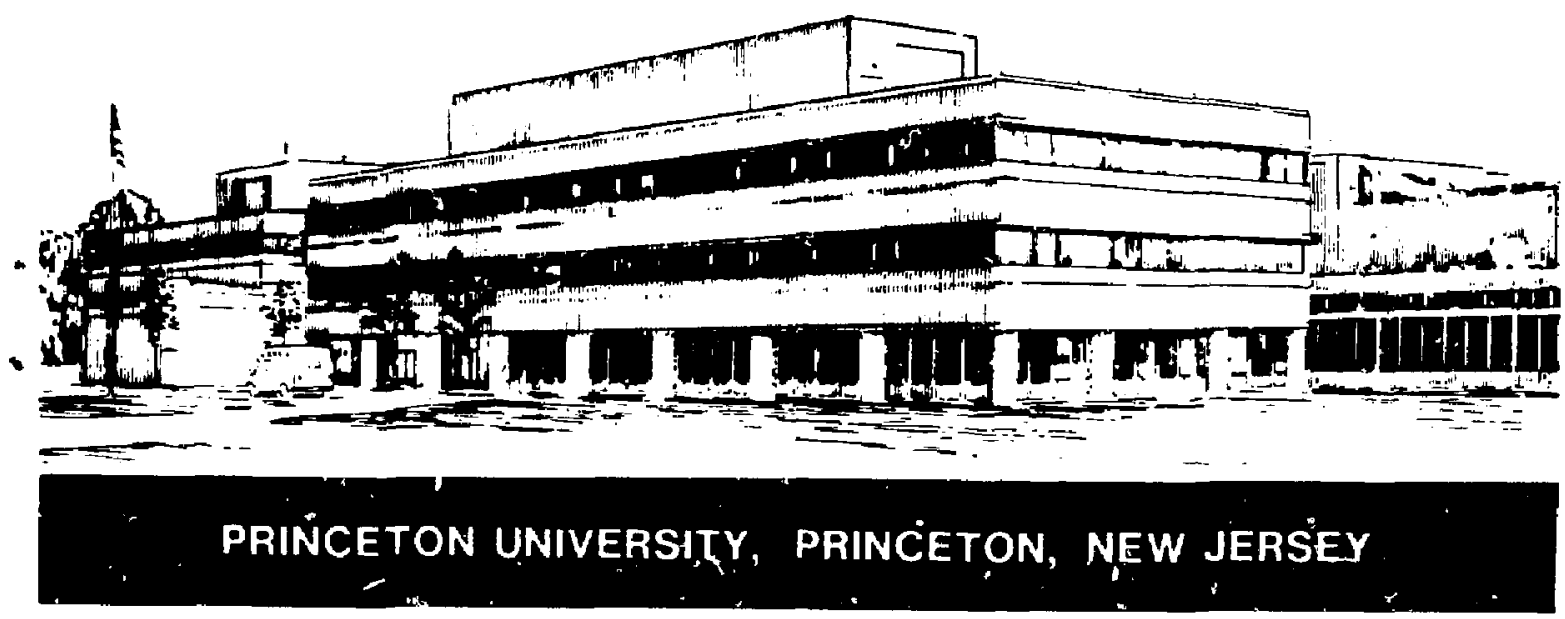




\section{NOTICE}

This report was prepared as an account of work sponsored by an agency of the United States Government. Neither the United States Government nor any agency thereof, nor any of their employees, makes any warranty, express or implied, or assumes any legal liability or responsibility for the accuracy, completeness, or usefulness of any information, apparatus, product, or process disclosed, or represents that its use would not infringe privately owned rights. Reference herein to any specific commercial produce, process, or service by trade name, trademark, manufacturer, or otherwise, does not necessarily constitute or imply its endorsement, recommendation, or favoring by the United States Government or any agency thereof. The views and opinions of authors expressed herein do not necessarily state or reflect those of the United States Governinent or any agency thereof.

\section{NOTICE}

This report has been reproduced directly from the best available copy.

Available to DOE and DOE contractors from the:

Office of Scientific and Technical Information P.O. Box 62

Oak Ridge, TN 37831;

Prices available from (615) 576-8401.

Available to the public from the:

National Technical Information Service

U.S. Departmient of Commerce 5285 Port Royal Road

Springfield, Virginia 22161

$70.3-487-4650$ 
PPPL -2760

DE91 013457

\title{
Tokamak Error Fields and Locked Modes
}

Presented as an Invited Talk at the Nov. 1990 APS Meeting

\author{
A. Reiman and D. Monticello \\ Princeton Plasma Physics Laboratory \\ Princeton, N. J. 08543
}

\begin{abstract}
The effects of externally applied, resonant magnetic field perturbations on tokamak plasmas are discussed. An analytical model assuming tokamak ordering and narrow islands clarifies the conditions under which error fields are amplified or diminished by self-consistent effects. A strong amplification of error fields near marginal tearing stability is found to broaden the range of parameters over which deleterious tearing effects are seen. Three-dimensional numerical solutions indicate that saturated tearing mode island widths can be sensitive to the presence of small nonaxisymmetric perturbatiors. It is argued that the theoretical conclusions are consistent with observations in ertor compensation experiments, and are also consistent with observations of enhanced growth for locked modes.
\end{abstract}


In this paper we discuss the effects of small, nonaxisymmetric magnetic field perturbations on tokamak plasmas. We focus on perturbations which can resonate with low order rational surfaces in the plasma and produce magnetic islands ( $m=n g$ for the poloidal and toroidal maie numbers $m, n$.) Error fields of this sort are produced in present day tokamaks by such things as finite tolerances in installation of the poloidal field coils, the presence of bus bars, etc. Typical magnitudes of such fields are $\delta B / B_{\mathfrak{q}} \approx 10^{-4}$, where $B_{t}$ is the toroidal field. Over the past few years, experimental evidence has accumulated that error fields of this magnitude can produce significant effects on tokamak discharges.

A coil which is capable of partially compensating the known error field bas been placed on DIII-D. This error compensation coil is believed to improve the general performance of the device. Iu particular, a systematic dependence of the low density locked mode threshold on the error compensation field has been documented. ${ }^{1}$

On Asdex, heat deposition on the divertor plates is nonaxisymmetric for If heated discharges, even in the absence of locked modes. The nonaxisymmetric deposition is believed to be due to the presence of islands produced by error fields. ${ }^{2}$

On JFT-2M, small fields produced by the ergodic limiter coil are found to systematically affect the threshold power for the L-H transition. ${ }^{3}$

In a number of tokamaks, rotating tearing modes are found to lock onto error fields. ${ }^{4,5}$ Locking exacerbates nonaxisymmetric heat deposition. It is also generally believed that locked modes disrupt more readily than rotating tearing modes.

These observations raise some serious concerns for the next generation of tokamaks. Heat load on the divertor plates is already an issue for these devices. Nonaxisymmetric heat deposition makes the situation worse by increasing the peak heat load. Also, the divertor plates can only tolerate a limited number of disruptions due to ablation of material from the divertor plates during disruptions. Disruptivity is therefore an issue.

In addition to the practical issues, error field effects also provide a testing ground for some interesting physics issues. A small, resonant magnetic field perturbation affects the plasma significantly only in a narrow region about the rational surface, allowing us to test our understanding of the physics of magnetic islands. We will also see that the presence of an infinitesimal non- 
axisymmetric perturbation provides a link between three-dimensional equilibrium theory and stability theory, suggesting an equilibrium approach to the computation of saturated tearing modes.

This paper will discuss several models for error field effects in tokamaks. We will start with some simple models to see what physics we can extract from them and what their limitations are. We will work our way up in complexity, and at the end will describe some three-dimensional numerical computations.

One widely used model for error field effects represents the plasma current by a single circular current filament. The vacuum field produced by this current filament is superposed on the vacuum error field to produce a model field, whose field lines are followed to determine flux surfaces and islands. For the known error fields in present day experiments, this typically predicts island widths which are a few percent of the minor radius. Although the calculated widths are probably not large enough to have a significant effect on a tokamak discharge, the widths predicted by this very crude model are sufficiently large to suggest that it is worth doing a more sophisticated calculation to determine the island widths more accurately.

The circular filament model neglects t'ae self-consistent response of the plasina currents to the presence of the error field. This response can be crudely incorporated in the model. At zeris $\beta$, currents follow the perturbed magnetic field lines. This can be modeled by distorting the circular filament to follow the magnetic axis. When this calculation was applied to nonaxisymmetric experiments on the ST tokanak, ${ }^{6}$ and on $\mathrm{PLT}_{1}{ }^{7}$ it was found that the computed island widths were greatly reduced from those calculated with a circular filament. The results suggest that any model with purely axisymmetric plasma currents is unreliable, that self-consistent currents can be important. The results have also been widely interpreted as showing that tokamaks are self-healing in the presence of error fields, that they always shield out nonaxisymmetric perturbations. (Although references 6 and 7 make no such claim.) We will see that this is not the case, that self-consistent plasma effects can amplify error fields.

There is at least one case where it is clear that an external perturbation will be amplified. If the profile is near manginal stability for tearing, the perturbation will feed off the free energy for the tearing mode. To see that this is so, we consider a sketch of a marginal tearing eigenmode in a cylinder (the 
lower curve in Fig. 1). The radial component of the field goes to zero at the boundary and at the magnetic axis, and it is finite in between. At marginal stability (zero growth rate), the tearing eigenmode is an equilibrium solution. A knowledge of the tearing eigenmode therefore allows us to determine what equilibria look like in the neighborhook of marginal stability. The upper curve in Fig. 1 is a sketch of an equilibrium solution, with an error field, just below marginal stability. It, too, must go to zero at the magnetic axis. At the edge, it has a finite value determined by the external perturbation. In between, it's shape must be close to that of the nearby marginal eigenmode. It is clear from this sketch that we get large amplification at the rational surface. (In contrast, the vacuun error field $B, \propto r^{m-1}$, where $m$ is the poloidal mode number.) In the absence of the error field, there would be no magnetic island below the tearing threshold. The presence of an error field effectively broadens the range of parameters over which we see deleterious tearing effects.

The filament model suggested that tokamak plasmas are sometimes selfhealing. We have argued that plasmas should amplify an external perturbation in some neighborhood of marginal teacing instability. We will now turn to a somewhat more sophisticated analytical model. One focus will be to gain a beiter understanding of the conditions under which we expect a tokamak plasma to be self-healing.

Our analytical calculation assumes narrow jslands and tokamak ordering. The solution for the perturbed helical fiux function putside the islands is determined by the tearing mode equation for the extesior region:

with the boundary condition

$$
\frac{d}{d r} r \frac{d \psi}{d r}-\frac{m^{2}}{r^{2}} \psi=\frac{j_{0}^{l} R}{B_{z}(t-n / m)} \dot{\psi},
$$

$$
\psi \longrightarrow \epsilon
$$

at the edge. ( $\epsilon$ is the given external perturbation.) This is the perturbed equilibrium equation. The perturbed current in the island, determined by Faraday's law, gives a jump condition across the island of the form

$$
r_{\nu} \psi^{\prime}\left(r_{0}\right)-r_{i} \psi^{\prime}\left(r_{i}\right)=I(w)
$$

where $r_{0}, r_{i}$ are the island edges and $\mathcal{I}(w)$ is related to the perturbed island current. The resulting model is a generalization of the usual nonlinear tearing 
mode theory ${ }^{8-10}$ to include the effects of a fixed external perturbation. Details of the calculation are presented in Ref. 11.

To make contact with the conventional $\Delta^{\prime}$ calculation, we let $\psi_{1}, \psi_{2}, \psi_{i}$ satisfy Eq. (1) with

$$
\begin{gathered}
\psi_{1}(r=a)=0, \quad \psi_{1}^{\prime}(r=a)=1, \\
\psi_{2}(r=a)=1, \quad \psi_{2}^{\prime}(r=a)=0, \\
\psi_{i} \longrightarrow r^{m} \text { as } r \longrightarrow 0 .
\end{gathered}
$$

We write

$$
\psi= \begin{cases}a_{1} \psi_{1}+\epsilon \psi_{2} & \text { for } r>r_{a}, \\ a_{i} \psi_{i} & \text { for } r<r_{i} .\end{cases}
$$

Applying the jump condition across the island, we obtain an eigenvalue equation for $w$ of the form:

$$
\alpha w^{3}-\Delta^{\prime} w^{2}= \pm \sigma^{\prime} \epsilon
$$

where

$$
\sigma^{\prime} \equiv \frac{4}{\psi_{0}^{\prime \prime}}\left[\psi_{1}^{c}\left(r_{0}\right) / \psi_{1}\left(r_{0}\right)-\psi_{2}^{\prime}\left(r_{0}\right) / \psi_{2}\left(r_{0}\right)\right] \psi_{2}\left(r_{o}\right)
$$

and where the \pm sign corresponds to the phase of the solution. In this expression, measures the stiength of the perturbation at the boundary (the error field). The coefficient $\Delta^{\prime}$ is the usual parameter measuring the freeenergy available to drive tearing

$$
\Delta^{\prime}=\psi_{1}^{\prime}\left(r_{s}\right) / \psi_{1}\left(r_{s}\right)-\psi_{i}^{\prime}\left(r_{s}\right) / \psi_{i}\left(r_{s}\right)
$$

where $r$, corresponds to the rational surface. The tearing mode is unstable for $\Delta^{\prime}>0$, and stable for $\Delta^{\prime}<0$. The $\alpha w^{3}$ term is primarily determined by the nonlinear modification of $\Delta^{\prime}$ in the presence of an island.

Figures 2 and 3 show schematically what the roots of the eigenvalue equation look like without and with an error field. The quantity $\psi\left(r_{s}\right)$ is plotted as a function of a profile parameter, with $\Delta^{\prime}$ assumed to increase monotonically as a function of the parameter. We plot $\psi$ at the rationa] surface rather than $w$ to retain the phase information.

Figure 2 shows the solution in the absence of an error field. For $\Delta^{\prime}<0$ there is a unique solution, corresponding to a stable, cylindrically symmetric equilibrium. For $\Delta^{\prime}>0$, the cylindrically symmetric equilibrium is unstable. 
There are also solutions corresponding to saturated tearing modes. These are stable, nonsymmetric equilibria with islands. As $\Delta^{\prime} \rightarrow 0$ from the right, the island width goes to zero. The point $\Delta^{\prime}=0$ corresponds to a bifurcation point of the solution.

When $\epsilon>0$, the solution of Eq. (2) is as shown in Fig. 3. There is still a unique, stable solution for $\Delta^{\prime}<0$, but the equilibrium is now a nonsymmetric one with an island. For large $\Delta^{\prime}$ there are again three solutions. The external perturbation breaks the bifurcation at $\Delta^{\prime}=0$, so that the solution now consists of two independent branches. The solution for $\Delta^{\prime}<0$ now goes smootbly into the locked mode solution for $\Delta^{\prime}>0$. This is the branch that we are interested in.

One thing that the picture of Figure 3 suggests is that a natural way to numerically compute the locked mode solution in three dimensions is to use a three-dimensional equilibrium code to track the solution in the presence of a nonaxisymmetric perturbation as we go from the stable regime to the unstable one. That is our approach to the calculation of locked modes. The picture of Figure 3 also has implications for the calculation of saturated tearing modes, even in the absence of an error field. The conventional approach to the calculation of saturated tearing modes, indicated in Fig. 4a, is to perturb an unstable axisymmetric equilibrium and use a time-dependent code to follow the tearing mode as it grows and saturates. If the saturated tearing mode is desired for several different values of the plasma parameters, the calculation is repeated for a corresponding number of different equilibria. The alternative approach indicated in Fig. $4 \mathrm{~b}$ is to apply a smail nonaxisymmetric perturbation and follow the equilibrium solution from the tearing stable regime into the unstable regime. This gives a direct calculation of the satu* rated tearing mode for a range of parameters. If desired, the finite $\epsilon$ solution for a given value of the profile parameter can be tracked to $\epsilon=0$, as indicated in the figure.

One thing that we would like to understand about the solution of Fig. 3 is whether the plasma amplifies or diminishes the error field when $\Delta^{\prime}<0$. To get a feeling for that, we conside: a special case where things simplify somewhat. We assume that the island is in the vacuum region outside the plasma. We get

$$
\sigma^{\prime}=\frac{8 m}{\psi_{0}^{\prime \prime} r_{s}} \frac{1}{\left(r_{g} / a\right)^{m}-\left(r_{s} / a\right)^{-m}}, \quad \alpha=0
$$


where we impose the error field at $r=a$. This gives an explicit expression for the island width,

$$
w^{2}=-16 \frac{\epsilon}{\psi_{0}^{\prime \prime}}\left(\frac{r_{2}}{a}\right)^{m} \frac{\Delta_{v}^{\prime}}{\Delta^{\prime}}
$$

where

$$
\Delta_{v}^{\prime}=\frac{2 m}{r_{*}} \frac{1}{\left(r_{d} / a\right)^{2 m}-1}
$$

is $\Delta^{\prime}$ for $j_{0}^{\prime} \rightarrow 0$ in Eq. (1). For $\Delta^{\prime}=\Delta_{\nu}^{\prime}$, we get the usual error field in vacuum. We can get amplification or self-healing, depending on whether $\left|\Delta^{\prime}\right|$ is greater than or less than $\left|\Delta_{v}^{\prime}\right|$.

The result is intuitively plausible. When $\Delta^{\prime}$ is sufficiently large and negative, the plasma is self-healing. When $\Delta^{\prime}<0$ approaches zero, we get amplification. There is a critical value of $\Delta^{\prime}$ at which the island width is just that due to the vacuum error field. As a particular example, for an $m=2$ island with $r_{*} / a=.3, \Delta_{v}^{\prime} \approx-13$.

Our formula for $w, \mathrm{Eq}$. (3), gives $w \rightarrow \infty$ as $\Delta^{\prime} \rightarrow 0$. This is because we have assumed that the island lies outside the plasma. There is no redistribution of curreat in the island as it increases in width. As $\Delta^{\prime} \rightarrow 0$, we approach marginal stability for the external kink. For an island in the plasma interior, the width remains finite as $\Delta^{\prime} \rightarrow 0$ due to the $\alpha w^{3}$ term in Eq. (3). The island width gets large in this limit, $w \propto \epsilon^{1 / 3}$ rather than $\epsilon^{1 / 2}$.

We turn now to numerical calculations. The focus here will be the unstable regime. We present here only a few illustrative calculations. A forthcoming paper will present an extensive set of numerical computations. ${ }^{12}$

As discussed above, we use a three-dimensional equilibrium code for our numerical calculations. We solve the three-dimensional equilibrium equation,

$$
\nabla_{p}=\mathbf{j} \times \mathbf{B},
$$

using the PIES code, ${ }^{13,14}$ which is capable of handling islands and stochastic regions.

As for any equilibrium solution, we need to specify two free functions to determine the pressure and current profiles. We specify a pressure profile, $p(\rho)$, and a quantity we will call $\mu$,

$$
\mu(p) \equiv\left(\mathrm{j} \cdot \mathrm{B} / B^{2}\right\rangle
$$


where $\rho$ is a coordinate that is constant on flux surfaces, and an average over a flux surface. The code allows stochastic regions and islands in the solution, and it flattens the profiles in those regions. The profiles on the good flux surfaces must also be adjusted to insure that there are no discontinuities at the edges of the flattened regions. To handle the profile adjustments, we define $\rho$ itself to be constant in the islands and stochastic regions. We define $\rho$ on the midplane by $d \rho / d R=1$ on the good surfaces, and $d \rho / d R=0$ in the islands and stochastic regions, where $R$ is the major radius. We extend $\rho$ off the midplane by taking it to be constant on the flux surfaces. As the code iterates, the widths of the islands and stochastic regions adjust self-consistently. At each iteration, a diagnostic in the code automatically determines where those regions are. The coordinate $\rho$ is then recalculated, and the profiles are correspondingly adjusted.

The calculations we show all have zero $\beta(p=0)$. We use the current profiles of Furth et al, ${ }^{15}$

$$
j(\rho)=j_{0}\left[1+\left(r / r_{0}\right)^{2 \gamma}\right]^{-(1+1 / \gamma)} .
$$

These current profiles have been used ext/nsively for tearing mode studies. We take $r_{0} / a=.6$, and we choose $j_{0}$ so that $\mathrm{q}$ on axis is 1.05 .

In addition to specifying the profiles, we also need to give a boundary condition. We specify the normal component of $\mathbf{B}$ on a bounding reference surface. This field can be calculated from a specified set of three-dimensional coils outside the bounding surface.

Although we can solve the fully three-dimensional problem in a torus, it is useful to also look at some solutions in a cylinder. Such solutions are helpful in understanding the physics, because the island width is tied directly to the radial component of the field $\left(w \propto\left|B_{7}\right|^{1 / 2}\right)$. We will look first at helical solutions in a long, thin cyinder $(R / a=20)$ for an $m=2, n=1$ mode, taking $\gamma=4$ in Eq. (4). The mode is unstable for our choice of parameters. Figure 5 shows the equilibrium value of $R_{0} B_{r} / a B_{o}$ as a function of $r / a$ for several different values of $B_{r}$ at the edge. The curve with $B_{r}=0$ at $r=a$ corresponds to the saturated tearing mode in the absence of an error fieid. The corresponding normalized value of $B_{r}$ at the rational surface is $1.6 \times 10^{-4}$, which gives an island width of about $5 \%$. In addition to $B_{r}=0$ at the edge. we also show cases with normalized $B_{r}$ values of $10^{-4}$ and $3 \times 10^{-4}$ at the edge. In the absence of a self-consistent plisma response, $B_{r}$ would decay 
linearly away from the boundary. For $R_{0} B_{r}(r=a) / a B_{\phi}=10^{-4}$, for example, the vacuum error field would have a magnitude at the rational surface about half that of the saturated mode. Instead, we find that the self-consistent plasma response strongly amplifies the error field, so that the field at the rational surface is about 5 times that at the edge, and about 3 times what it is in the absence of an error field. Multiplying the field at the edge by a factor of 3 gives little change in the shape of the eigenfunction, so that the field at the rational surface is now about 4 times what it is at the boundary, and about 8 times what it is in the absence of an error field.

Now we turn to some toroidal, fully three-dimensional solutions. Figures 6 and 7 show Poincare plots for solutions with $R / a=3$ and $\gamma=1$, without and with an error field. (We caution that the origin of our coordinate system does not lie on the magnetic axis, so the region about the axis looks like an island, but is not an island.) The tearing mode is unstable for these parameters. In the absence of an error field there are saturated islands at the $q=2$ and $q=3$ rational surfaces, with the total island width across the midplane about $6 \%$.

The solution in Fig. 7 has an error field imposed with resonant $n=1$ and $n=2$ Fourier components. The magnitude of the Fourier components were calculated from a small shift and elliptical deformation of a nearly circular poloidal field coil at $R=6 a$. The coil current and shift were chosen to approximately reproduce the reported $n=1$ error field spectrum on DIII-D, with an $n=1, m=2$ amplitude of about $5.5 \times 10^{-5} B_{t}{ }^{1}$ (The shift is $0.5 \%$, and the current is approximately that required by the Shafranov formula for radial force balance. ${ }^{16}$ ) The elliptical deformation was also taken to be $.5 \%$. This is comparable to the maximum measured elliftical deformation of the TFTR poloidal field coils. With the imposed error field, the islands are now larger and more numerous. The total island width across the midiplane is now about $23 \%$.

Finally, we discuss the correspondence between our theoretical results and some of the experimental observations.

The DIIl-D tokamak encounters a locked mode at low density. The density threshold for this mode has been found to depend systematically on the field in the error compensation coil. In particular, using the error compensation coil to reduce the ambient error field makes: it possible to reach lower densities. This behavior is consistent with our conc!usion that the presence of 
an error field broadens the range of plasma parametes's cver which deleterious tearing effects are secn.

On a number of tokamaks, loched modes are believed to grow to larger amplitude and to disrupt more readily than rotating modes. This is also something tha : our calculations wnuld lead us to expect. When a magnetic isiasd is rotating rapidly past an error field, the growth of the island is not strongly affected by the preserce of the error field. Induced wall rurrents can slow the mode rotation, and allow ihe mode to lock onto the external error field. ${ }^{17}$ The ertor field can then strongly affect the saturation amplitude, and therefore the suhsequent evolution, of the stationary island. The absence of wall stabilization also entance the growth of loclied modes. ${ }^{18}$ The relative importance of these tiwn effects has yet to be sorteri out. Measurements of the toroidal plasma rotation on $\mathrm{JET}^{19}$ and Asdex ${ }^{5}$ indicate that it is globilly damped to a small valua $i$. the presence of locked modes, su that flow effects can be safely -aglected in calculating the subsequent evolution of the mode.

We conclade tha: experime it and theory are presenting us with a consistent pictu se indicating that erroi fieids of the magnitude that exist in present day tokarkaks ar having sigrificant effects. The theory predicts that error fields can be sirongly : nplified below the tearing threshold. It follows that the presence of error felds broadens the range: of plasma parametery over which deleterious tearing effects are seen. The theory also predicts that the saturation amplitude of tearing modes can be sensitive to the presence of relatively small error fields. These theoretical results suggest that error field amplitudes in the next generation of tokamak experiments will affect heat deposition peaking factors on the divertor plates (througl nonaxisymmet. tic heat deposition), and will affect disruptivity. Improved understancing of error field effects will play a role in the design of the next generation of tokamaks in terms of suggesiing construction tolerances, and pussibly in terms of surgesting the desurability of trim coils for tining out residual field errors.

We are indebteo' to R. Goldston, N. Pomphrey, W. Stodiek, and L. Zakharov for useful discussions. This work was supported by the United States Department of Energy under Contract DE-AC02-76-CHO-3073. 


\section{References}

${ }^{1}$ T. Scoville et al., Bull. APS 31, 2118 (1989).

${ }^{2}$ T. E. Evans, J. Neuhauser, F. Leuterer, E. R. Mueller, and the Asdex Team, Characteristics of toroidal energy deposition asymmetries in Asdex, Technical heport GA-A20076, General Atomics, 1990.

${ }^{3}$ A. W. Leonard et al., Bull. A PS 35 (1990).

4J. A. Snipes et al., Nucl. Fusion 28, 1085 (1988).

${ }^{5} \mathrm{H}$. Zohm, A. Kallenbach, H. Bruhns, G. Fussmann, and O. Klueber, Europhys. Lett. 11. 745 (1990).

${ }^{6}$ W. Stodiek, K. Bol, H. Eubank, S. V. Goeler, and D. J. Grove, Effect of non-axisymmetric confining fields on tohamak discharges, in Plasma Physics and Controlled Nuclear Fusion Research, Proceedings of the Fourth In.Lernational Conference, Madison, Vol. I, pages 465-478, 1971.

${ }^{7} R$. Goldstion et al., Confinement studies during neutral beam injection in PLT, in Heating in Toroidal Plasmas, pages 711-721, Commission of the Furopen Communities, 1980, Proceedings of the 2nd Joint GrenobleVarenna International Symposium - Villa Olmo - Como - Italy.

${ }^{8}$ R. B. White, D. A. Monticello, M. N. Rosenbluth, and B. V. Waddell, Phys. Fluids 20, $\$ 00$ (1977).

${ }^{9}$ R. B. White, Rev. Mod. Phys. 58, 183 (1986).

${ }^{10} \mathrm{~L}$. Zakharov, Quasilinear equilibria related to tearing modes in tokamaks, Technical Report FRCR 319, Fusion Research Center, University of Texas, Austin, Texas, 1988.

${ }^{11}$ A. Reiman, Tokamak magnetic islands in the presence of nonaxisymmetric perturbations, to be published.

${ }^{12}$ A. Reiman and D. Monticello, Sensitivity of tokamck equilibria to the presence of nonaxisymmetric error fields - numerical computations, to be published, 1990 . 
${ }^{13}$ A. H. Reiman and H. S. Greenside, Comput. Phys, Commun. 43, 157 (1986).

${ }^{14}$ A. H. Reiman and H. S. Greenside, jcp 87, 349 (1990).

${ }^{15}$ H. P. Furth, P. H. Rutherford, and H. Selberg, Phys. Fluids 16, 1054 (1973).

${ }^{16}$ V. D. Shafranov, Reviews of Plasma Physics, volume 2, pages 103-151, Consultants Bureau, New York, 1966.

17M. F. F. Nave and J. A. Wesson, Mode locking in tokamaks, in Controlled Fusion and Plasma Physics (Proc. 14th Eur. Conf. Madrid, 1987), Vol. 11D, Pt. III, page 1103, European Physical Society, 1987.

${ }^{18}$ M. Persson and A. Bondeson, Nucl. Fus. 29, 989 (1989).

${ }^{19}$ J. A. Snipes, D. J. Campbell, T. C. Hender, M. V. Hellermann, and H. Weisen, Nucl. Fusion 30, 205 (1990). 


\section{Figures}

FIG. 1. Sketch of a marginally stable tearing eigenmode (lower curve) and an equilibrium solution just below the tearing threshold (upper curve).

FIG. 2. Schematic plot of $\psi\left(r_{s}\right)$ as a function of a profile parameter for $\epsilon=0$. We assume that $\Delta^{\prime}$ increases monotonically with the profile parameter.

FIG. 3. Solution for $\dot{\psi}\left(r_{s}\right)$ with finite $\varepsilon$. The dashed line indicates the solution in the $\epsilon=0$ limit.

FIG. 4. Schematic view of saturated island calculation by a) conventional time-dependent methods, and b) tracking the equilibrium solution.

FIG. 5. Normalized radial field for a helical equilibrium solution with several different values of the error field.

FIG. 6. Poincare plot for a toroidal solution with $R / a=3, \gamma=1$ and no error field.

FIG. 7. Poincare plot for a toroidal solution with $R / a=3, \gamma=1$ and error field as described in the text. 
$90 \mathrm{~T} 1003$

Equilibrium, with error field,

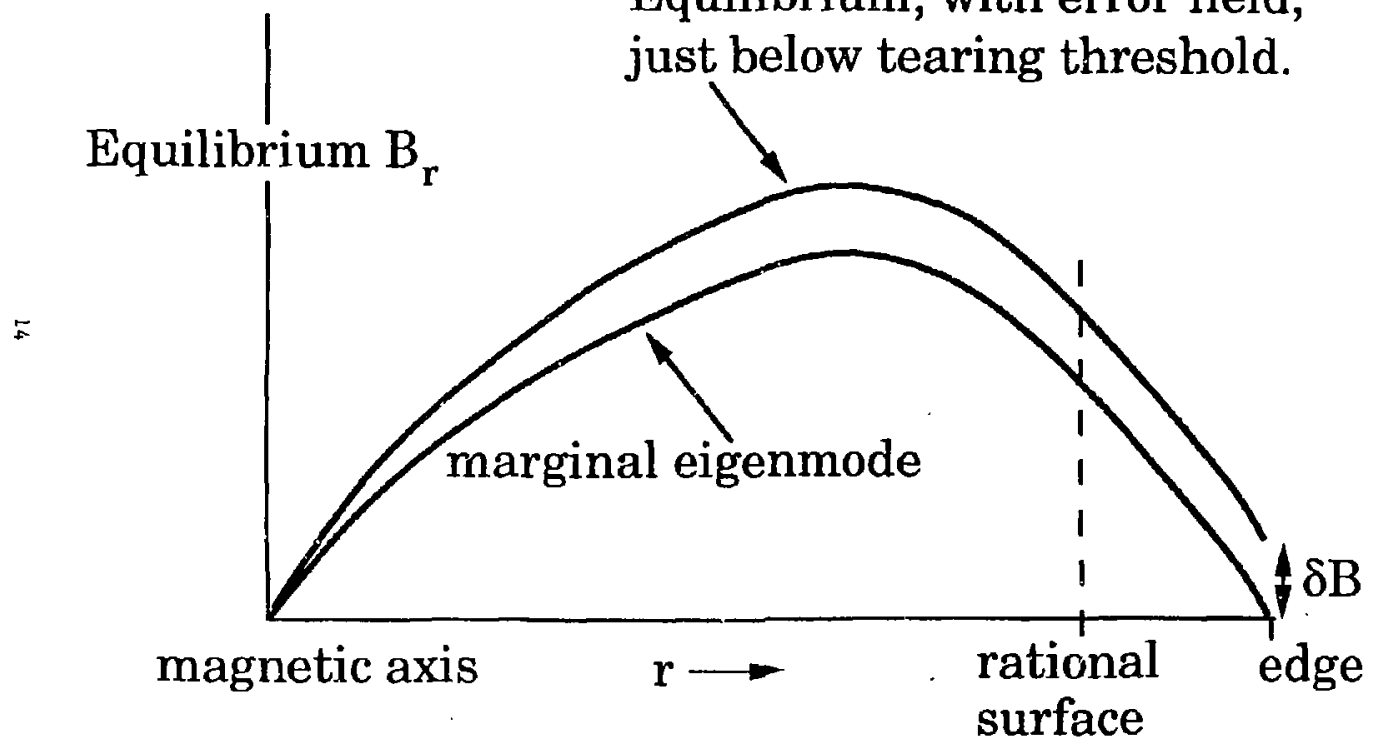

F1g. 1 


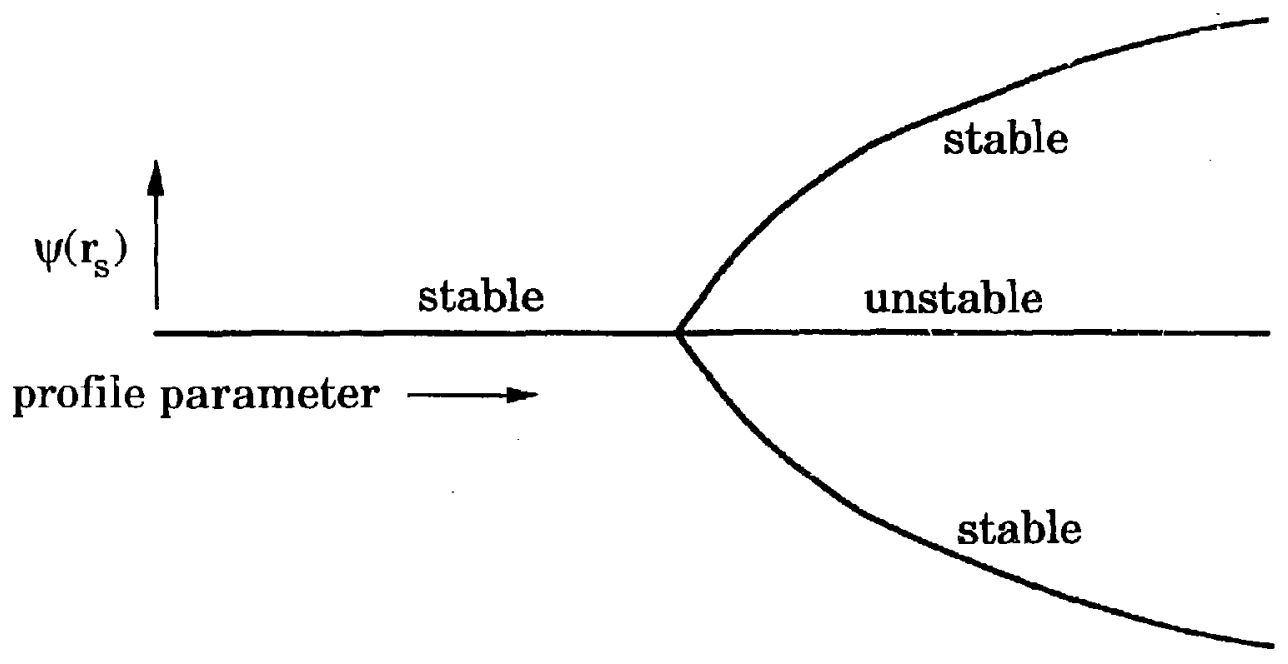


$90 T 1005$

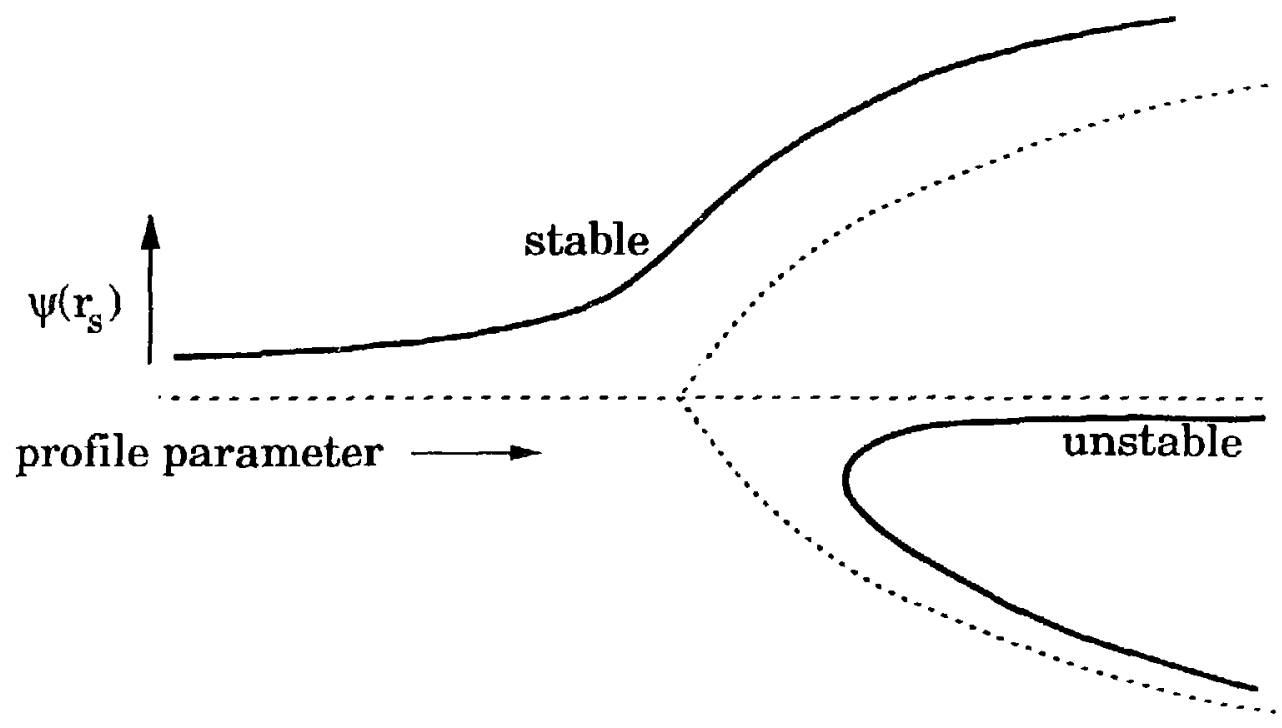


a) conventional time-dependent calculation

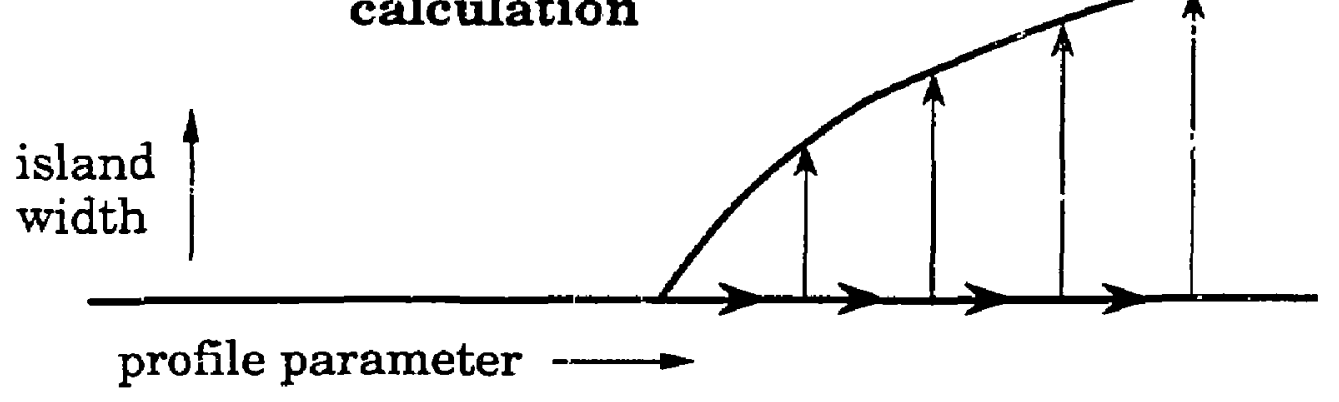

b) equilibrium calculation

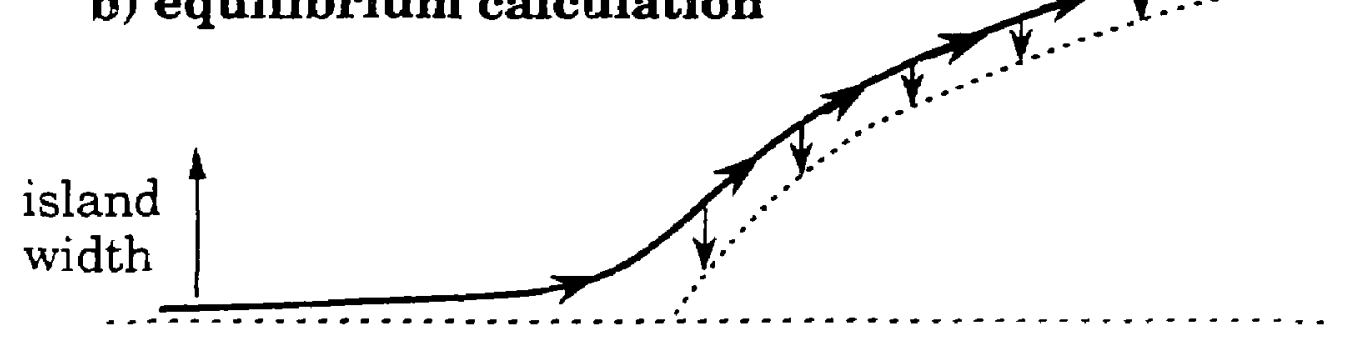
profile parameter

Fig. 4 
$90 T 1007$

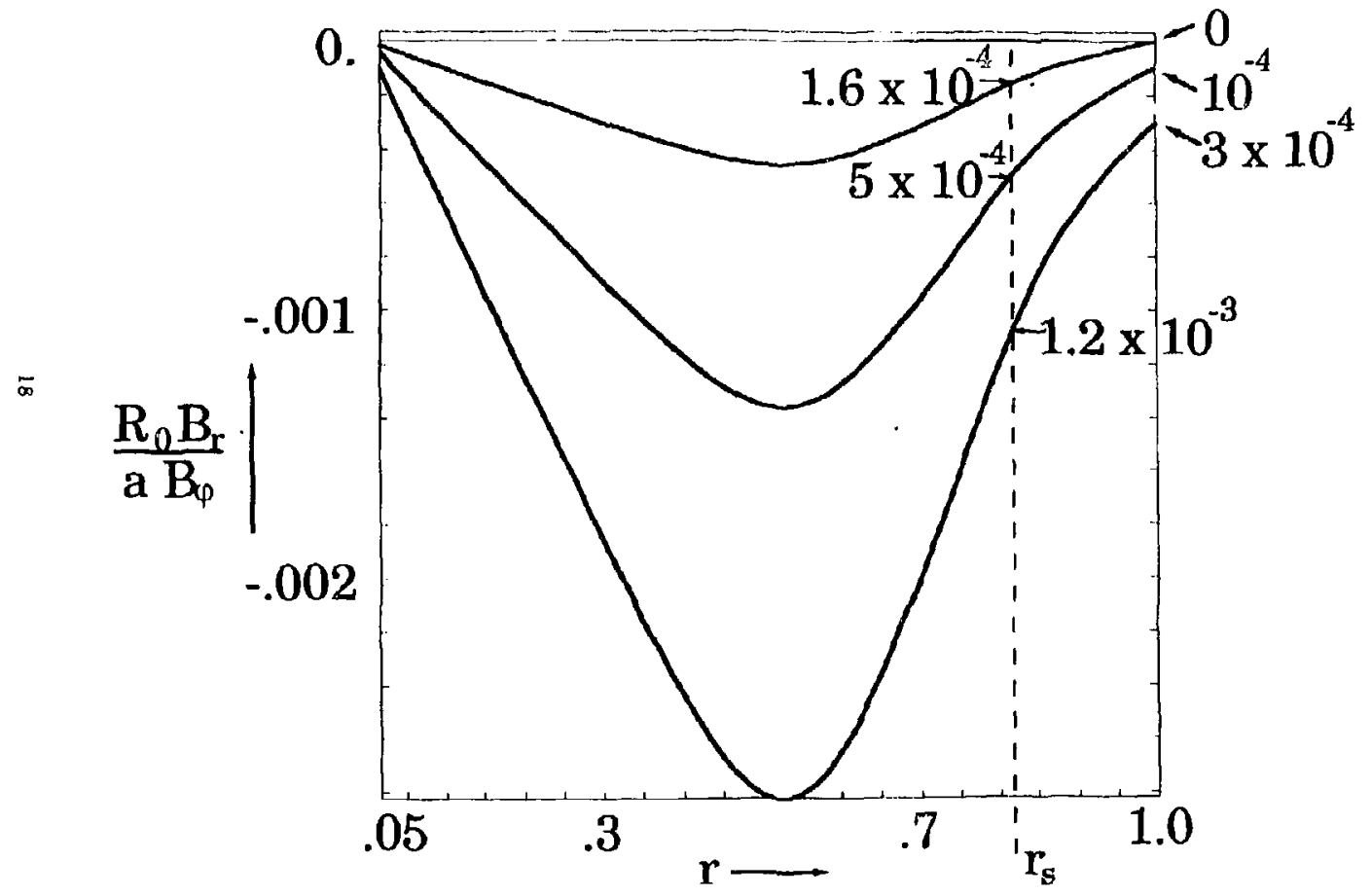

Fig. 5 
$90 \mathrm{~T} 1008$

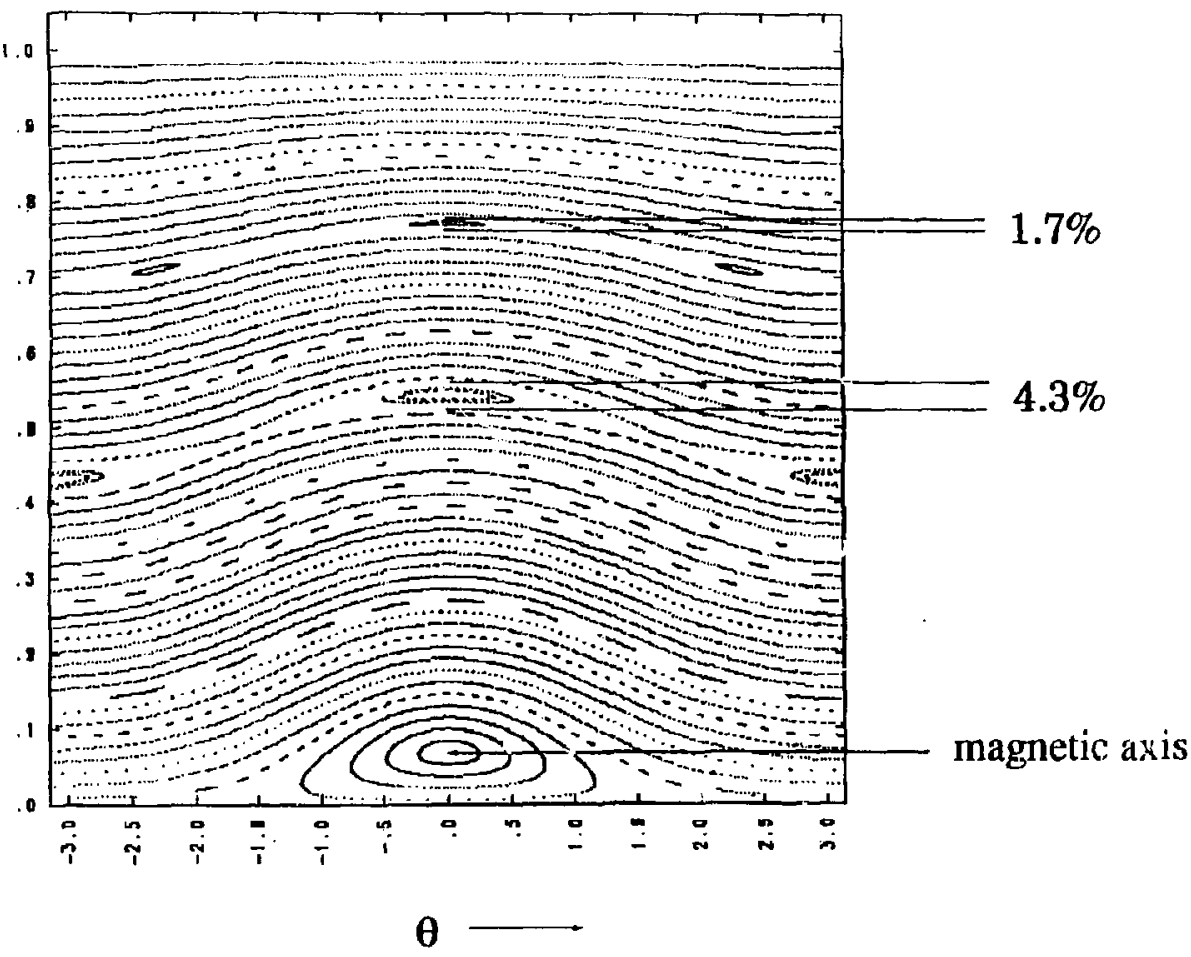

F1E. 6 
$90 \mathrm{~T} 1009$

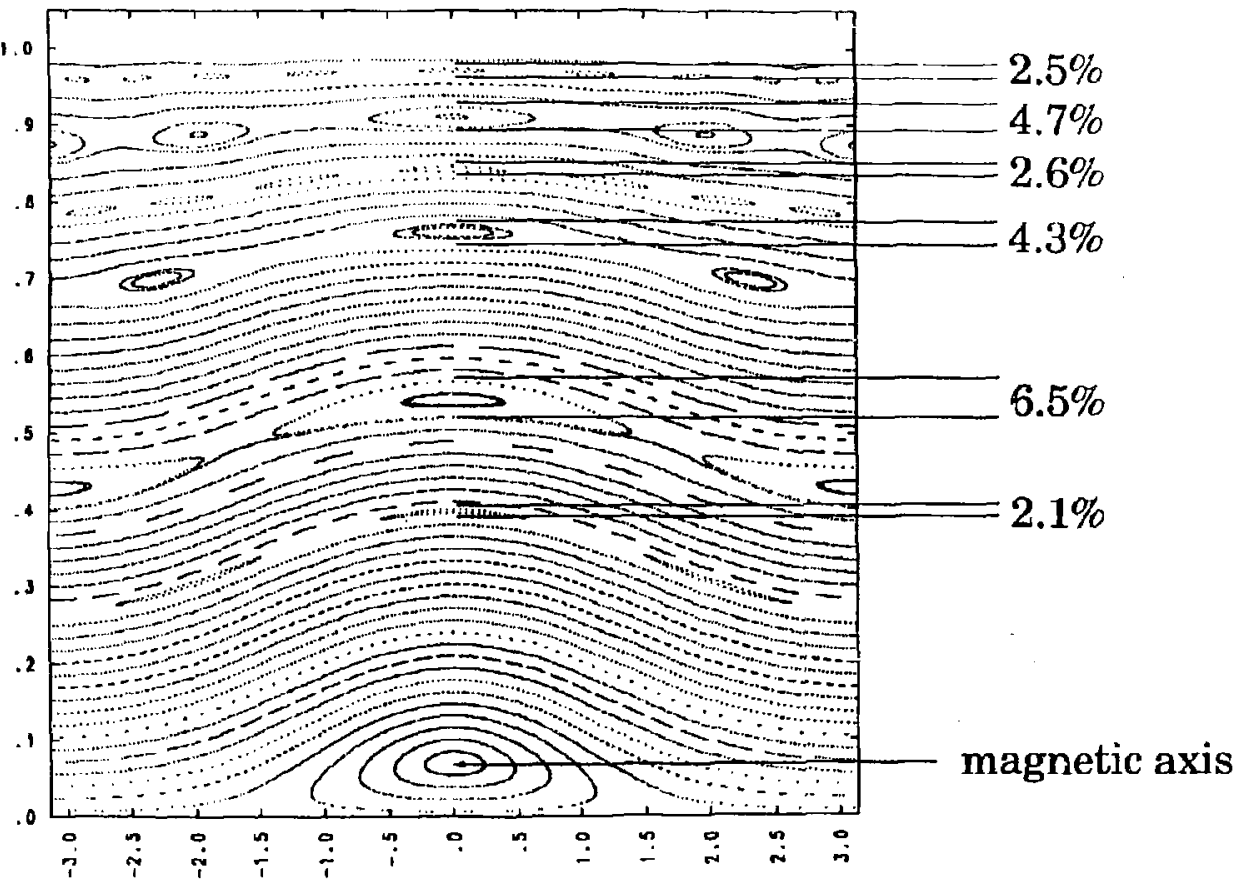

$\theta$

F1g. 7 